\title{
MCCN: A Softwarized Approach to 5G
}

\author{
Sławomir Kukliński ${ }^{1}$, Khoa Truong Dinh ${ }^{2}$, Tinku Rasheed ${ }^{3}$
}

\begin{abstract}
G networks are expected to be deployed by 2020 and 5G is currently a hot research topic receiving considerable attention from industry and academia alike. It is expected that the latest networking technologies like Software-Defined Networking (SDN), Network Function Virtualization (NFV) and Mobile Edge Computing will play a pivotal role in the design of the integrated 5G network infrastructure. This paper shows how the mentioned technologies (baptized together as network softwarization) may impact the 5G networks design, operations, standardization and business models. A high level concept of Mobile Code based Convergent Network (MCCN), based on a dynamic placement of software modules in a distributed cloud environment, that is able to cope with $5 \mathrm{G}$ requirements will be discussed in detail and we argue the potential benefits of the proposed solution.
\end{abstract}

\section{Index Terms - Softwarization, SDN, 5G, cloud, NFV}

\section{Introduction}

The mobile networks evolve at a very fast pace. Originally the main goal of mobile networks was to provide voice services. Recently the situation has evolved significantly and the dominant traffic in mobile networks is the IP traffic originated due to the huge influx of mobile applications. It is predicted that this traffic will grow exponentially in the future, so appropriate capacity of mobile systems will have to be provided. The mobile networks evolve not only in order to fulfil significantly increased traffic demands and the increasing number of applications/users, but also to improve service agility and to simplify and automate their deployment and management.

Standards developed by 3GPP (part of ETSI) [1], so- called Releases, are important milestones in the evolution of mobile networks. These releases are used not only to deploy new mobile network generations (like UMTS, LTE), but also to make incremental modifications of the existing ones, and were used in the past in order to introduce solutions such as GPRS, EDGE, HSPA and LTE-A. It is worth to mention that in the past sixteen years, eleven 3GPP Releases have been issued what means that the average time between Releases is about 17 months. Such fast technology update is troublesome for operators, which have to upgrade the installed solutions in relatively short periods of time. This problem is crucial when the deployment of a new 3GPP release requires hardware upgrade and much less troublesome when it deals with software upgrade only. This is the first reason that shows the

\footnotetext{
${ }^{1}$ Sławomir Kukliński was with the Faculty of Electronics and Information Technology, Warsaw University of Technology, Poland and Orange Polska

${ }^{2}$ Khoa Truong Dinh was with the Faculty of Electronics and Information Technology, Warsaw University of Technology, Poland

${ }^{3}$ Tinku Rasheed was with the CREATE-NET, Italy
}

importance and benefits of software-based solutions in mobile networks.

At present the 3GPP is working on the Release 13 [2] as an upgrade to $4 \mathrm{G}$ networks, but the most recent objective is to define the requirements for $5 \mathrm{G}$ networks, in order for the technology to be mature to be deployed by 2020. Similar activities have just started in USA, Japan, South Korea and China [3-7].

The 5G research directions are still evolving but their common denominator is to use the so-called network softwarization paradigm. Network softwarization is relatively a new term. It is often related to the use of technologies like network virtualization, clouds, NFV (Network Function Virtualization) and SDN (Software-Defined Networking). These technologies can be nicely combined together. Network softwarization may change significantly the way in which networks are built and operated. Such expectation is based on so far unseen flexibility, programmability, openness and expected cost reduction by using generic hardware and specialized software for $5 \mathrm{G}$ network implementation. An exciting possibility is fast and dynamic deployment of network functions/services in an on-demand style. It is expected that software-based networks will shorten the timeto-market, enable easy service personalization and require much more relaxed and faster standardization than hardware based solutions. The software approach is also key enabler for nodes and network virtualization. Moreover, as it was already mentioned, that the software based upgrades are in general easy, therefore it can be expected that continuous system evolution will be easily supported in the softwarized 5G.

In this paper a high level concept of $5 \mathrm{G}$, called Mobile Code based Convergent Network (MCCN), based on a dynamic placement of software modules in a distributed cloud environment, will be outlined. It will be also shown that the software technologies, like those used in MCCN, can significantly change the way in which the $5 \mathrm{G}$ networks are designed and operated. The CAPEX costs of such approach can be significantly reduced due to the multifunctional (in the context of Fixed Mobile Convergence) role of hardware and software platforms used for the deployment of $5 \mathrm{G}$ networks. The proposed approach is able to fulfil the technical and economical requirements posed by the NGMN [5] and 5G PPP [4] industry associations.

The rest of the paper is organized as follows. In Section II the requirements and design considerations for $5 \mathrm{G}$ networks are presented. In Section III the already proposed concepts to use software-based technologies for $5 \mathrm{G}$ like SDN, NFV and clouds are discussed. In Section IV the MCCN concept is presented and its impact on 5G standardization and business model is discussed. Finally, Section V summarizes the paper. 


\section{5G requirements and design considerations}

The work on $5 \mathrm{G}$ has started since 2014 by the definition of the expectations and high-level requirements regarding this mobile network. In Europe a set of main $5 \mathrm{G}$ requirements has been developed by 5G PPP [4], and another long list of requirements has been provided by NGMN [5]. It is important to recall some of 5G PPP key requirements, because of their impact on 5G network design. Another important facet regarding the design of $5 \mathrm{G}$ networks is the identification of appropriate use cases and business models for 5G. The METIS project [3] has developed a set of potential use cases that could be ideal business enablers for a $5 \mathrm{G}$ mobile network. According to the 5G PPP, the 5G network should, in comparison to $4 \mathrm{G} \mathrm{LTE}$ as of 2010 :

- handle 1000 higher traffic volume,

- provide 10-100 higher user's bit rate,

- decrease about 5 times delays (to ca. $1 \mathrm{~ms}$ ),

- provide extremely energy efficient operations,

- $\quad$ provide faster service deployment,

- handle simultaneously much more terminals per base station (Internet of Things [IoT] in mind),

- $\quad$ provide zero perceived downtime.

According to the NGMN, it is also expected that $5 \mathrm{G}$ will be cost effective (in term of both, OPEX and CAPEX).

The abovementioned requirements impact the $5 \mathrm{G}$ system design:

- In order to cope with the high base stations capacity and user traffic demands, the radio channel has to be wide and therefore very high frequencies (so-called mm-waves) will have to be used. Actually there is on-going research related to the air interface, which works at $12 \mathrm{GHz}$ or 60 $\mathrm{GHz}$ channels [8-10]. The usage of such high frequencies reduces the useful cell size significantly. The number of cells required to cover a certain area will have to be very high what leads to the so-called Ultra Dense Network (UDN). UDN raises many issues related to mobility handling (huge number of handovers), it requires dense and high capacity fronthaul links and implies high overall cost of the radio access elements in the network. Moreover, some Radio Air interface Technologies (RATs) require the synchronization of radio transmissions of several radio stations (cf. CoMP - Coordinated MultiPoint), which implies centralized control plane of RAT. Further, there is a probability that because of the specific requirements of IoT (Internet of Things), several RATs will be used simultaneously in $5 \mathrm{G}$;

- In order to minimize the transmission delays and to avoid bottlenecks the data plane should be distributed as much as possible. Moreover all the control plane operations have to be very fast.

- In order to minimize energy consumption special techniques related to dynamic energy management of the under-utilized nodes and efficient reuse of the networking resources has to be applied.
- In order to provide fast service deployment, the NFV orchestration can be used.

- In order to handle thousands of IoT devices, the control traffic for IoT transmission has to minimized.

- In order to provide extremely high reliability, a fault tolerant system design has to be applied.

In the context of mobile network softwarization, it is important to analyse the mobile networks architecture evolution. In fact, in current mobile systems, the generic approaches to telecommunications system design were followed. In such approach, the architecture is split into the data plane, control plane, and management plane. Such functional split is of the utmost importance in the context of softwarization. In the management and control planes, transactions are typically handled and the management plane traffic is usually much less intensive than the control plane traffic. In the data plane, traffic is intensive and typically handled by specialized hardware. Design of broadband data plane using software requires significant computing power. On the contrary the management plane is already implemented in software. The control plane can be implemented in software, however in existing mobile networks, the control plane is not programmable. The latest trends and some requirements (including $5 \mathrm{G}$ requirements) show that, in order to minimize the transmission delay and to avoid network bottlenecks the data plane should be distributed as much as possible, on the other hand the control plane operations should be logically centralized in order to better optimize network resources and to synchronize certain radio operations. Such concept can be relatively easy to implement if separated network nodes are used as data plane and control plane devices. A nice example of such concept is currently studied in 3GPP Release 13, which enables direct communication between terminals (D2D communications) [2][30]. In the D2D concept only the data plane links between terminals are direct, whereas D2D signalling is obtained from the base stations.

It is therefore agreed that softwarization techniques are the potential solution to fulfil $5 \mathrm{G}$ requirements and expectations. In that context, MCCN has been proposed in this paper as a candidate of the softwarization approach to $5 \mathrm{G}$.

\section{Present softwarization approaches to mobile networks}

There are already proposed $5 \mathrm{G}$ designs which assume the use of software-based approaches like SDN, NFV, clouds and Mobile Edge Computing (MEC) [28].

\section{A. SDN in mobile networks}

SDN is a data networking paradigm created in 2008 [16]. The main idea of this concept lies on separation at the device level between control and data planes. The separation is provided by the specification of so-called Southbound protocols (e.g., the OpenFlow protocol). The control plane of SDN is logically centralized and the network controller is responsible for all network operations, especially it controls directly the way in which data flows are forwarded. In opposite to existing IP networks, SDN operations are focused not on packets but on flows. The SDN network controller 
Proc. of The Third Intl. Conf. on Advances in Computing, Control and Networking - ACCN 2015.

Copyright (C) Institute of Research Engineers and Doctors, USA .All rights reserved.

ISBN: 978-1-63248-082-8 doi: 10.15224/ 978-1-63248-082-8-05

provides an interface (Northbound Interface) to applications that gives the ability to set data forwarding rules by applications. SDN is at present widely used in all cases where dynamic traffic redirection is necessary (it includes inter-cloud communication [shown in Fig. 3], NFV, etc.). There are also some approaches that demonstrate profits of using SDN in case of mobility handling. In [17] it has been proposed to use the SDN controller for simultaneous control of radio and nonradio nodes and it has been shown that due to the use of SDN the number of control messages needed to handle handover in comparison to LTE X2 interface based handovers can be reduced. A similar approach is evaluated in FP7 project CROWD [19] and in [18]. SDN can be used as a single logical entity to control RAN and Core elements of the network. In such case no strict separation between RAN and the core part of the network exists. In this approach there is a possibility to obtain high distribution level of the data plane which solves well-known problems of fronthaul and backhaul traffic congestion.

\section{B. NFV in mobile networks}

NFV [20] is a new networking paradigm, which enables virtualization of node functions. This virtualization lies on the usage of generic servers (or clouds) and virtual machines in order to deploy virtual networking functions (VNFs). ETSI NFV has defined MANO (Management and Orchestration) Framework [21], which enables dynamic deployment of VNFs using NFV Infrastructure in a seamless manner. This process is driven by the so-called NFV Orchestrator. In some interpretations the main benefits of NFV are related to the functionality upgrade of the installed node and to rapid service deployment. Both cases are important in the context of 5G, however much more interesting case is a dynamic deployment of VNF in geographically distributed data centres (clouds, edge clouds) in an on-demand style. In fact, it is expected that NFV solutions will be based on clouds and more discussion on their usage in $5 \mathrm{G}$ will be provided in subsequent sections.

\section{Clouds for mobile networks}

A very special and highly popular case of software-based 5G is C-RAN (Cloud-RAN, Centralized-RAN). This concept has been originally introduced by IBM [22] and from 2010 is intensively developed by China Mobile [23]. In this concept the 'hardware-defined' functionality is very limited. The main idea of C-RAN is to use very simple antenna nodes (called RRH, Remote Radio Heads) connected to a single cloud by fibres (see Fig. 1). For the purpose of RRH-cloud connection the well-known CPRI (Common Public Radio Interface) interface is used [24]. The CPRI interface is an industry standard interface developed in 2003 (the new versions however were developed in recent years) and already used by many vendors in their $3 \mathrm{G}$ or $4 \mathrm{G}$ RAN solutions. Using this interface, sampled I/Q radio signals are synchronously transmitted from base station sectors to the controller of these RRHs, named the Broadband Unit (BBU), which is responsible for the processing of the signal and transferring processed data to the core part of the network. Vendors can change the functionality of BBU to be specialized software (i.e. implement new 3GPP Releases, even to convert
UMTS/NodeB into LTE/eNB). In C-RAN there is no BBU as a hardware node but all RRHs are directly connected to a datacentre that hosts the software based BBU pool. Due to the use of single mode fibres the RRHs can be placed far away from the cloud - because of technical constraints this distance should be however shorter than $40 \mathrm{~km}$. C-RAN should be able to handle hundreds of even thousands of RRHs. There are significant benefits of the proposed approach that explains the popularity of this concept. The first benefit is the enormous flexibility of the system. The functionality can be fully defined in software i.e. the same hardware solution can be used for GSM, UMTS, LTE or 5G, as long as the RRH supports appropriate radio channels. All RAN related operations are performed in software that concerns all planes of the system. This solution therefore can be upgraded efficiently. Moreover, there is no explicit core part of this network, it is just replaced by a cloud-based platform. In fact, C-RAN can be seen as a single base station with huge number of antennas. This interpretation shows another benefit related to mobility handling - all handovers are handled in the same cloud, therefore making it a very fast process. The simplicity of RRHs makes the management of C-RAN very simple. Centralization of control operations enables the usage of radio synchronized operations like CoMP etc. Another benefit of the approach is the aggregation of all resources in the cloud that provides high level of their reusability. In classical mobile networks $(2 \mathrm{G}, 3 \mathrm{G}$ or $4 \mathrm{G})$ distributed RAN nodes handle certain geographic areas and due to variable time-of-day users' behaviour some nodes approach high load during business hours only and are under-utilized afterwards. In CRAN such behaviour concerns only a certain set of RRHs, because the cloud platform handles the users independently on their location. That way much better reusability of control and data planes resources is achieved. In C-RAN the energy consumption can be reduced if the mechanism of switching off of the unused RRHs is deployed. It has been shown that CRAN provides $70 \%$ [23] better usage of BBU resources than the classical case with distributed BBUs. Some simulations have shown that a similar gain can be achieved in term of energy savings [25].

Unfortunately, C-RAN has also several significant drawbacks. The most important one is very inefficient and centralized transmission of data between RRHs and BBUs using CPRI which is a synchronous interface that transmits sampled I/Q radio signals. Unfortunately, the amount of the transmitted data is independent on the users' traffic. In case of LTE, the traffic from a single, 3 sectors cell is in range of 16 Gbps [26]. The CPRI transmission requires in fact a dark fibre to be implemented. There are right now on-going activities in order to compress CPRI data for transmission or to use Ethernet for CPRI transmission, but it seems that the gain obtained that way cannot be significant. The centralization of the data plane, that exists in C-RAN is also an important problem in terms of the end-to-end delay minimization. The CPRI transmission in a $20 \mathrm{~km}$ fibre takes $100 \mu$ s one way [23] and such delay can be troublesome in certain radio related operations leading also to relatively small areas to be handled by C-RAN - it cannot provide countrywide coverage. Moreover, a serious problem may happen when two or more C-RAN based solutions will have to handle inter-C-RAN 
handovers. So far there are no standardized architecture or work on multiple C-RANs.

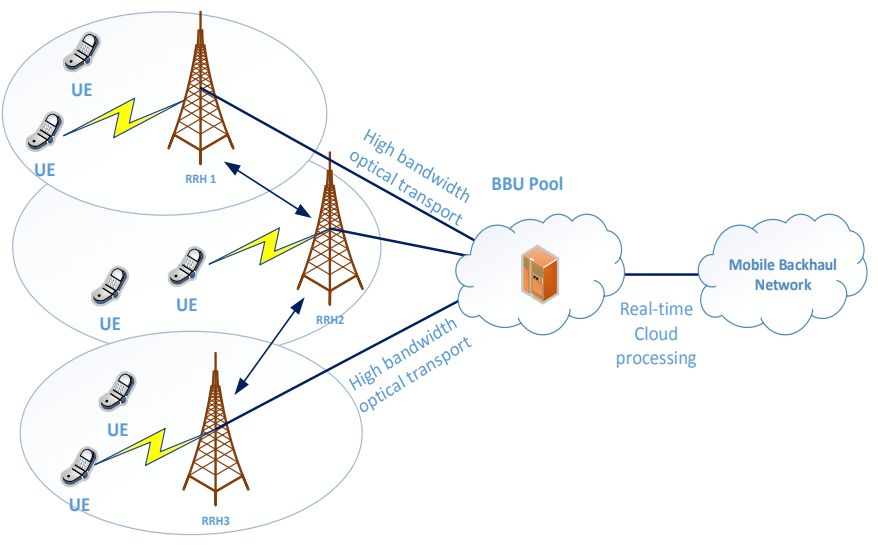

Figure. 1. The C-RAN concept

Target applications for C-RAN include medium or small cities or special areas like stadiums. Despite its drawback there are already some experiments and C-RAN trials all over the world [23].

\section{Research projects on softwarized mobile networks}

There are already some research projects that propose the usage of networks softwarization for evolved $4 \mathrm{G}$ and $5 \mathrm{G}$ networks.

The SoftNet project [11] decentralizes the mobile network architecture and comprises of SDN based core network and unified RAN. By deploying SDN and NVF, SoftNet is able to dynamically enable required virtual network functions and deploy new network mechanisms.

SoftRAN [12] argues that the distributed control plane in current LTE system is suboptimal in dense wireless deployment. They proposed a software-defined centralized control plane for RAN that abstracts many local base stations into a virtualized big-station.

MobileFlow [13] describes the Software Defined Mobile Network architecture built on the decoupling of data plane and control plane in mobile network. This architecture enables operators to orchestrate new service chains and evolve their networks by updating/replacing various mobile applications. OpenRan [14] provides Software Defined RAN virtualization. By extending the SDN capabilities to handle wireless networks, it achieves network nodes' virtualization and the programmability of the network. The concept provides programmability of both, control and data planes, making that way the RAN flexible and open.

Kempf et al. [15] have provided extensions to the OpenFlow protocol specification (ver. 1.2) to allow flow routing for GTP (GPRS Tunnelling Protocol), so EPC (Evolved Packet Core) functional entities can be decoupled from network elements (such as S-GW, PDN) and moved to virtual machine in the data centre.

\section{Iv. The MCCN concept}

\section{A. The MCCN architecture overview}

The analysis of the above-mentioned approaches and research projects linked with $5 \mathrm{G}$ has led to the conclusion that so far there is no appropriate solution that provides high flexibility, efficient usage of resources and is able to cope with the $5 \mathrm{G}$ requirements [5]. The most advanced concept, i.e., the C-RAN approach provides excellent flexibility and resource re-usage but it is ineffective and expensive. A single C-RAN is not able to cope with a big geographical area due to transmission range limitations mentioned in the previous section. Moreover, the $5 \mathrm{G}$ requirement to reduce the transmission delay to $1 \mathrm{~ms}$ can be a limiting factor for the usage of this technology for C-RANs with long CPRI links. It is however possible to create a network that is a combination of many C-RAN-like networks. This is the main concept on which of the Mobile Code based Convergent (MCCN)

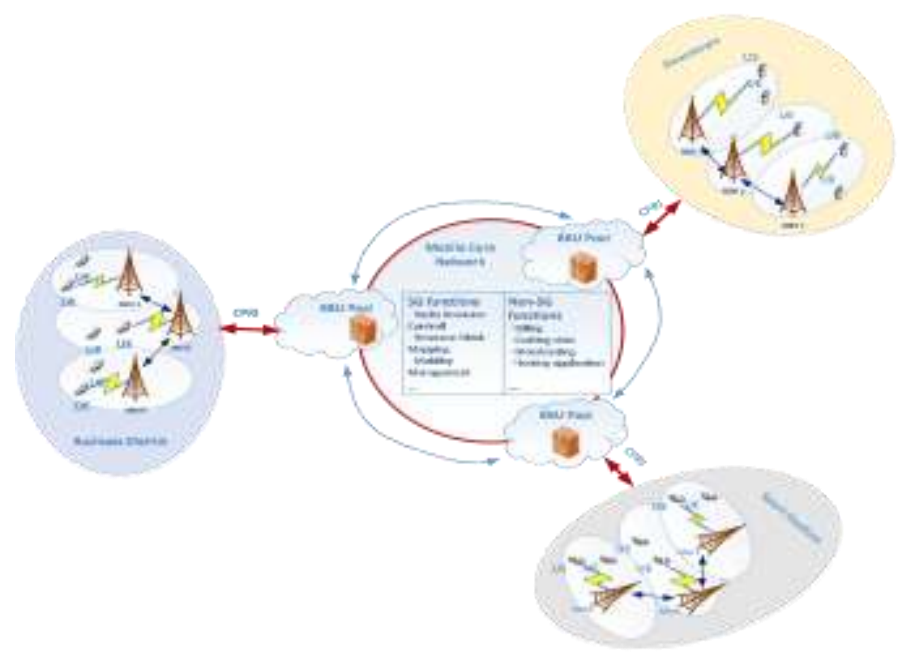

Figure. 2. The MCCN architecture

network (see Fig. 2) is based upon.

The MCCN network hardware architecture is composed of several distributed and interconnected edge clouds and attached to them, via CPRI interfaces, RRHs and other access networking access solutions like FTTx, or xDSL. Each of the edge clouds is used to instantiate telco cloud based applications including the $5 \mathrm{G}$ functions. Due to such $5 \mathrm{G}$ as a Service (5GaaS) approach and using the NFV framework for the deployment of highly granular $5 \mathrm{G}$ functions it is possible to instantiate as many control or data plane $5 \mathrm{G}$ functions that are needed to handle dynamically changing traffic and user demands in different geographic areas. A relevant feature that can be implemented in MCCN is a seamless shift of some 5G functions from one edge cloud to another one in the followthe-crowd approach. Such mechanism should support seamless code mobility that provides continuity of active sessions. 
Proc. of The Third Intl. Conf. on Advances in Computing, Control and Networking - ACCN 2015. Copyright (c) Institute of Research Engineers and Doctors, USA .All rights reserved. ISBN: 978-1-63248-082-8 doi: 10.15224/ 978-1-63248-082-8-05

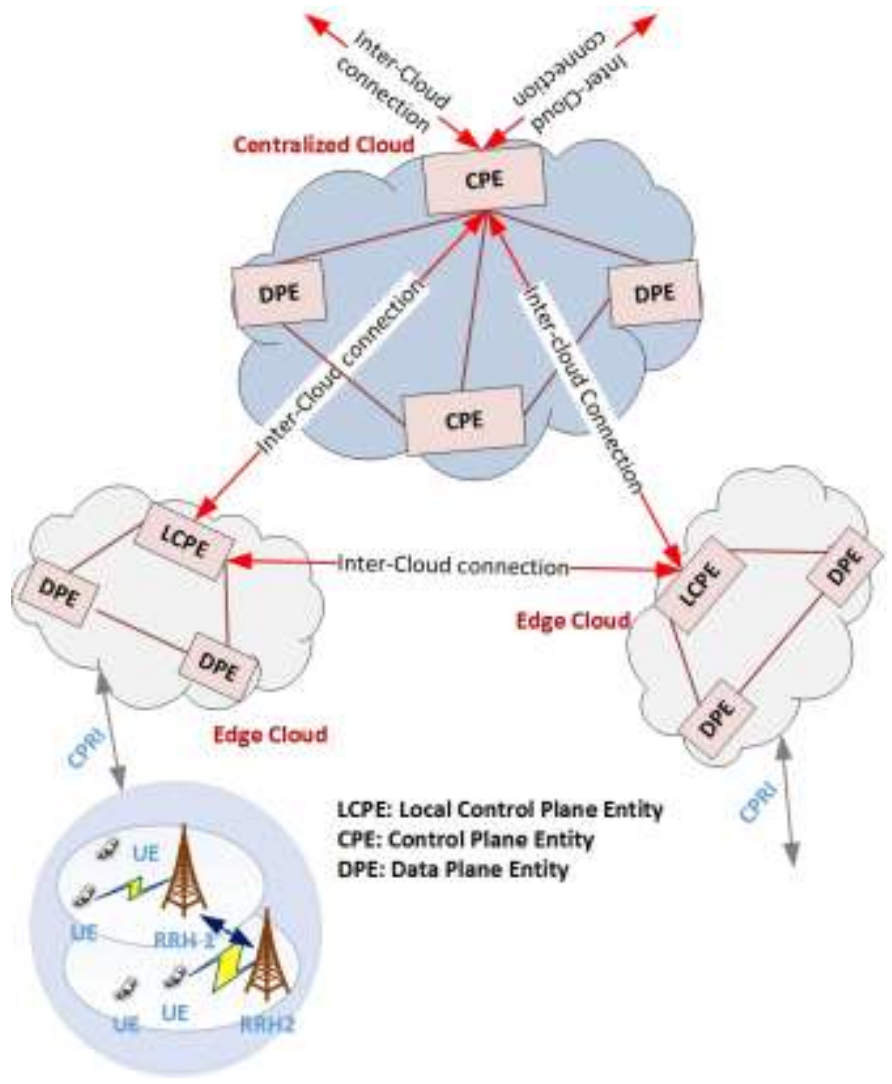

Figure. 3. The software architecture of MCCN (an example)

In MCCN there is proposed a centralized control plane and multi-cloud based distributed data plane. Due to such approach MCCN provides similar benefits like those provided by C-RAN (global network view), but the distributed data plane enables much lower delays of the transmission and shortens inefficient CPRI based transmission.

In order to reduce 5G CAPEX and to increase cloud resources efficiency the clouds used for $5 \mathrm{G}$ can and should be also used for other purposes. They can be for example used as intelligent and programmable nodes which are a part of fixed line networking solutions playing the role of so-called Pointsof-Presence (POP), caching data, broadcasting data locally or hosting applications for intelligent homes or offices. The clouds can be also used at night, for performing some other commercial tasks, for example to create billing reports etc. That way high reusability of the computing and storage resources can be provided. Such approach provides very efficient integration of fixed and mobile networking solutions (converged access, integration of networks with applications) significantly reducing 5G related CAPEX and OPEX.

The fronthaul cost of MCCN is significantly reduced in comparison to C-RAN; in the proposed approach the edge clouds are placed closer to the RRHs than in the C-RAN case what reduces the overall fronthaul length (see Fig. 3). Moreover, the same optical fibres cables that are used for fronthaul (typically composed of 12 or even 24 fibres) can be used for the deployment of FTTB or FTTH solutions. That way the CAPEX related to $5 \mathrm{G}$ fronthaul deployment can be split among many fibre-based access technologies.
It is worth to note that the on-going work on micro-clouds [32] nicely supports the proposed MCCN idea reducing the cost and footprint of edge clouds.

\section{B. MCCN implementation details}

It is expected that the implementation of MCCN software functions (in a $5 \mathrm{GaaS}$ style) will be based on the enhanced NFV MANO framework. The enhancement should consists of some extensions related to software modules seamless mobility capability with the preservation of their 'activity states'. The software modules of high functional granularity will be defined as separate modules (VNFs according to NFV) for the data and control plane, which will enable different placement of both planes software modules, i.e. more distributed data plane modules placement and centralized placement of the control plane modules. In the context of MCCN the distribution means that the data plane software components will be placed in every edge cloud, whereas the control plane software module can be responsible for controlling of data planes entities which belongs to multiple or even all edge clouds in the network. For the sake of the performance and for increased network reliability some control plane modules can be implemented on per cloud basis. The 5G software modules of data plane of each clouds should be responsible for the physical layer processing (demodulation, framing), link layer processing and basic scheduling. Due to some advanced data plane operations like coordinated scheduling the virtualization can't be implemented as data plane function only. In case of MIMO, and CoAMP the appropriate processing should be implemented as a part of the data plane but it has to be supported by appropriate control plane modules installed in the same cloud in which the controlled data plane entities are. The data plane functions supported by appropriate control plane functions should expose a virtualized network view that is needed for multi-tenant and multi-service networks.

The logically centralized 5G control plane should include all the functions that are at present realized by LTE control plane entities, i.e., MME (i.e. mobility management, resource management, authentication), PCRF (accounting, QoS management) and HSS (master database of users and their service profiles). Their centralization or decentralization at the certain level (delegate of some control plane functions or some functions) in order to improve the system performance and reliability needs more detailed analysis. The instantiation of the control plane entities and their placement should be based on 'mobile traffic hotspots' localization and on the needed control plane performance. The instantiation of $5 \mathrm{G}$ data plane modules is depended on the local (i.e. at the cloud level) demands and can be used as a mechanism to implement energy efficient operations, i.e. switching off some RRHs and software modules responsible for their handling during offpeak hours.

The localized VNF management and on-fly orchestration can be achieved leveraging the edge cloud platforms that can be globally orchestrated using a centralized hypervisor platform. Leveraging the edge cloudification will also permit Mobile Service Providers to leverage on network data effectively to enable big data oriented applications [29]. 
There is no doubt that the overall optimization of the MCCN based network is a complex real-time process and appropriate adaptive and intelligent (i.e. cognitive) algorithms will have to be used for that purpose. Such operations should be implemented as MCCN management which should be programmable and able to cope with the dynamic, software based functionality of the network. An important part of the MCCN network management is an orchestrator that is responsible for the dynamic and seamless deployment of new network functions and services and for the seamless relocation of already active modules.

The detailed specification of software modules that compose this $5 \mathrm{GaaS}$ is to be defined yet and is not critical in terms of system deployment - the hardware part can be deployed in advance and used for other purposes, i.e. fixed access network, data caching and so on. An important issue is however related to the APIs of every software module.

In MCCN the inter-cloud networking and topology is managed through SDN. SDN provides multiple profits in that context. First, SDN provides much more efficient networking solution than CPRI (aggregation of the users' traffic), the mobility in SDN can be handled more efficiently than in IP networks [17], last but not least SDN is a perfect tool for traffic redirection necessary for the dynamic deployment of the NFV functions. Moreover, the applications or operator policy rules can easily change the way in which data flows are forwarded in the network.

In conclusion the MCCN concept lies on the usage of multifunctional edge clouds for the implementation of all 5G functions in software like in C-RAN (possibly using the NFV framework) with the ability of on-fly shifting of some functions from one cloud to another one, on the use of the SDN technology for flexible and efficient intra and inter-cloud connections, and on the reuse of the fibre-based converged access for other technologies like FTTx.

\section{The impact of the MCCN concept on 5G standardization}

Standardization is quintessential in order to provide not only the interoperability of terminals made by different vendors, but also to provide interoperability of hardware network nodes produced by different manufacturers. Due to the long and complex equipment production process, standards have to be defined in advance. Later on, the implementation of network hardware nodes blueprints the detailed design (ASICs, printed circuit boards, hardware specific firmware). The whole process is costly, time consuming and the devices have low level of upgradeability.

Network softwarization adopted by the MCCN concept simplifies and shortens the standardization procedure. In software-based solutions the network functions are implemented as applications on already standardized (de facto or de jure) software platforms and generic hardware platforms. It is therefore possible to avoid detailed standardization of node functionality - the necessary functionality can be dynamically bootstrapped during network operations. A significant part of 5G mobile networks in MCCN defined as distributed cloud application.
There is no doubt however that the terminal radio interface has to be standardized, the same concerns the external interfaces to the management systems and applications as well as software modules APIs. It is worth to mention however that MCCN may profit from standardization works on SDN (OpenFlow), NFV and clouds.

\section{The impact of the MCCN concept on the $5 G$ business model}

Network softwarization used in MCCN may significantly change the way in which $5 \mathrm{G}$ networks will be deployed and operated. This change will concern both the vendors and the operators. First of all it is expected that $5 \mathrm{G}$ vendors will deliver software modules that will be responsible for specific $5 \mathrm{G}$ functions.

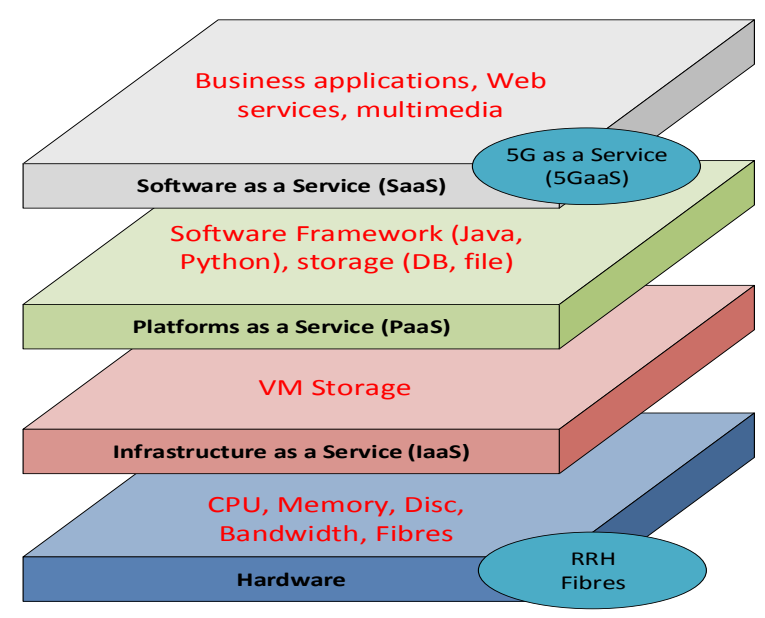

Figure. 4. 5GaaS (MCCN) generic decomposition [27]

The use of widely accepted open source platforms and software components should reduce software cost and increase interoperability. There is however a need for a business entity responsible for their proper integration. NFV-like, single cloud or edge cloud based 5G (like MCCN) solutions can be "decomposed" into (see Fig. 4) Infrastructure as a Service (IaaS), Platform as a Service (PaaS), Software as a Service (SaaS) and $5 \mathrm{G}$ as a Service (5GaaS) according to [27]. Such decomposition defines also different roles of business entities. IaaS or PaaS providers can treat $5 \mathrm{G}$ as one of many applications that are served by them, therefore the computing and storage resources can be used for many purposes, i.e. more efficiently than for $5 \mathrm{G}$ only. There is no doubt that in order to build $5 \mathrm{G}$, IaaS has to be complemented by $5 \mathrm{G}$ specific radio nodes (RRHs) with fibres that are used for their connection to 5G specific or (in case of $\mathrm{MCCN}$ ) multi-purpose clouds. If different entities will be involved in building of $5 \mathrm{G}$ infrastructure (in a broad sense) the total cost of ownership can be significantly reduced, the resources will be used in an optimum manner and the time to market will be shortened.

Having in mind that $5 \mathrm{G}$ will use a large number of very small cells (UDNs), the possibility of CAPEX reduction and to provide split business model is of premium importance. Multi-tenancy may benefit from MCCN based 5G by using the 
same, shared physical infrastructure. Such approach is much more efficient than building a $5 \mathrm{G}$ infrastructure by each mobile network operator.

\section{v. Concluding Remarks}

The development of $5 \mathrm{G}$ networks is on-going at very fast pace. The solutions are expected to be ready on the market by 2020. It is widely expected to build 5G using software solutions like NFV, cloud and SDN. It seems however that the impact of softwarization on $5 \mathrm{G}$ system design is underestimated [31]. Network softwarization can introduce much bigger revolution in the system design than it is estimated and will significantly impact $5 \mathrm{G}$ network design, operations and business models. The use of software technologies like NFV and SDN can significantly reduce the efforts related to the system design and implementation, moreover the standardization process can be significantly simpler and the deployment will be much more cost effective.

Very popular C-RAN concept has many valuable features, however due to the range constraints it cannot be deployed countrywide and multiple C-RANs in such case have to be deployed. A modification of C-RAN by the use of multiple edge clouds leads to MCCN, a new concept that we introduced in this paper. MCCN is a fixed-mobile convergent solution based on edge clouds. It reduces the delay of the transmission between RRHs and clouds and lowers the overall length of the fronthaul in comparison to C-RAN. The usage of clouds not only for $5 \mathrm{G}$ but also for other purposes increases the overall level of resource utilization and may speed-up 5G deployment. The edge cloud based implementation of $5 \mathrm{G}$ can fully reuse the NFV architecture with VNF responsible for 5G functions and other operations, but it can also go beyond that by using software techniques like mobile agents for network troubleshooting etc. There is no doubt that in MCCN the most important problem is related to efficient and fast inter-cloud procedures, like handover. This issue can be nicely solved by the use of SDN for inter-cloud connection and virtual machine migration mechanism in order to handle moving mobile groups of users to provide data and/or control plane load balancing.

Network softwarization is also a key enabler of network virtualization and can fundamentally change the mobile network operator landscape. The same may happen with mobile systems vendor's market.

It is worth to mention however that in order to make software based $5 \mathrm{G}$ network a reality, still many problems have to be solved yet. Some of them, like performance, security and high reliability of software are already well known.

\section{References}

[1] http://www.3gpp.org/specifications/releases

[2] 3GPP Rel.13, V0.0.6, 2014.06. http://www.3gpp.org/ftp/Information/ WORK_PLAN/Description_Releases/

[3] https://www.metis2020.com

[4] http://5g-ppp.eu/projects/

[5] https://www.ngmn.org/work-programme/5g-initiative.html

[6] http://www.imt-2020.cn/en/menu/all?cid=0\&mid=0\&type=1

[7] http://5gmf.jp/en/
[8] Rappaport, Theodore S., et al. "Millimeter wave mobile communications for 5G cellular: It will work!." Access, IEEE 1 (2013): 335-349.

[9] Roh, Wonil, et al. "Millimeter-wave beamforming as an enabling technology for $5 \mathrm{G}$ cellular communications: theoretical feasibility and prototype results." IEEE Com. Magazine 52.2 (2014): 106-113.

[10] Weiler, Richard J., et al. "Enabling 5G backhaul and access with millimeter-waves." European Conference on Networks and Communications, EuCNC. Vol. 2014. 2014.

[11] Wang, H., Chen, S., Xu, H., Ai, M., \& Shi, Y. (2015). SoftNet: A software defined decentralized mobile network architecture toward 5G. Network, IEEE, 29(2), 16-22.

[12] Gudipati, Aditya, et al. "SoftRAN: Software defined radio access network." Proceedings of the second ACM SIGCOMM workshop on Hot topics in software defined networking. ACM, 2013.

[13] K. Pentikousis, et al., "Mobileflow: Toward Software-Defined Mobile Networks," IEEE Communications Magazine., vol. 51, no. 7, Jul. 2013, pp. 44-53.

[14] M. Yang et al., "OpenRAN: A Software-Defined RAN Architecture via Virtualization," Proc. ACM SIGCOMM, Aug. 2013, pp. 549-50.

[15] J. Kempf, et al., "Moving the Mobile Evolved Packet Core to the Cloud," IEEE Wireless and Mobile Computing, Networking and Communications (WiMob), Oct. 2012, pp. 784-91.

[16] N. McKeown et. al "OpenFlow: enabling innovation in campus networks," Sigcomm Comp. Com., vol. 38, no. 2, pp. 69- 74, Mar 2008

[17] Kuklinski, Slawomir, Yuhong Li, and Khoa Truong Dinh. "Handover management in SDN-based mobile networks." Globecom Workshops (GC Wkshps), 2014. IEEE, 2014.

[18] Mahmoodi, T., \& Seetharaman, S. On Using a SDN-based Control Plane in 5G Mobile Networks, Wireless World Research Forum, meeting 32, Marrakech, Morocco, May 2014.

[19] Ali-Ahmad, Hassan, et al. "CROWD: An SDN Approach for DenseNets." Software Defined Networks (EWSDN), 2013 Second European Workshop on. IEEE, 2013.

[20] ETSI, "Network Functions Virtualization," White paper, Oct 2012 [Online]. Available: http://portal.etsi.org/NFV/NFV_White_Paper2.pdf.

[21] Mehmet Ersue, "ETSI NFV Management and Orchestration An Overview", http://www.ietf.org/proceedings/88/slides/slides-88opsawg-6.pdf

[22] Y. Lin, L. Shao, Z. Zhu, Q. Wang, and R. K. Sabhikhi, "Wireless network cloud: Architecture and system requirements," IBM Journal of Research and Development, january-february 2010.

[23] Mobile, China. "C-RAN: the road towards green RAN." White Paper, ver 2 (2011).

[24] "Common Public Radio Interface (CPRI); Interface Specification V6.0," August 2013.

[25] Checko, Aleksandra, et al. "Cloud RAN for Mobile Networks-a Technology Overview." (2014).

[26] J. Segel, "lightRadio Portfolio: White Paper 3," Alcatel-Lucent Bell Labs, Tech. Rep., 2011.

[27] Zhang, Qi, Lu Cheng, and Raouf Boutaba. "Cloud computing: state-ofthe-art and research challenges." Journal of internet services and applications 1.1 (2010): 7-18.

[28] ETSI, "Mobile Edge Computing", White paper [Available online] https://portal.etsi.org/Portals/0/TBpages/MEC/Docs/Mobileedge_Computing_-_Introductory_Technical_White_Paper_V1\%201809-14.pdf

[29] A. Bradai, T. Ahmed, T. Rasheed and K. Singh, "Cellular Softwaredefined Networks - A Framework" in Proc. IEEE Communications Magazine [in Press], June 2015.

[30] X. Lin et al., "An Overview of 3GPP Device-to-Device Proximity Services," IEEE Commun. Mag., vol. 52, no. 4, Apr. 2014, pp. 40-48.

[31] S. Kuklinski, "Underestimated Role of Software in 5G", IEEE International Workshop on Software Defined 5G Networks, [Keynote] London April 2015.

[32] Nakao, Akihiro. "Flare: Open deeply programmable network node architecture." (2012). 